\title{
Update on new biologics for intractable eosinophilic asthma: impact of reslizumab
}

This article was published in the following Dove Press journal:

Drug Design, Development and Therapy

Jagdeep Sahota'

Douglas S Robinson ${ }^{2}$

'Department of Respiratory Medicine, University College London, London, UK; ${ }^{2}$ Department of Respiratory

Medicine, University College Hospital NHS Trust, London, UK
Correspondence: Douglas S Robinson Calle Blanca 17, Dos Torres, 14460, Spain Email dsrobinson@allergyandasthma. co.uk

\begin{abstract}
A small percentage of patients with asthma have uncontrolled symptoms and frequent exacerbations, despite treatment with inhaled corticosteroids and other agents. It has become clear that different subtypes of this severe, treatment-resistant group exist due to different mechanisms of the disease. All such patients require detailed assessment in specialist centers to characterize the disease and assess treatment adherence. Recently, monoclonal antibodies have become available, which target specific pathways that may contribute to persistent inflammation and asthma exacerbations. These antibodies include those targeting interleukin (IL)-5, which drives eosinophilic inflammation. Reslizumab is a newly licensed antibody that blocks binding of IL-5 to its receptor. Here, we discuss the significance of clinical data of this drug, which show up to $50 \%$ reduction in exacerbation rates, together with modest but significant improvements in lung function and quality of life, in those with persistent eosinophilia. The combination of reslizumab with mepolizumab and benralizumab, which also target IL-5, may be a useful addition to the therapeutic armamentarium in a selected group of patients with severe asthma.
\end{abstract}

Keywords: asthma, eosinophil

\section{Introduction}

Asthma is a disease that affects 300 million people worldwide, with incidence increasing year-on-year. It is a heterogeneous condition supported by the fact that one treatment approach does not "cure all" and explains the variations in presentation. This is due to the complex nature of the underlying immune dysregulation that occurs in the disease and the interplay between the innate and adaptive immune systems as well as genetic and environmental factors. ${ }^{1}$

The current management has largely centered on increasing the intensity of treatment in a stepwise manner from short-acting inhalers at one end through to inhaled corticosteroids (ICS) to systemic corticosteroids and biologics at the other end in order to achieve control of symptoms. ${ }^{2,3}$ Although this approach can be effective in most patients, adherence to long-term use of inhaled preventer medication is typically only $\leq 50 \%$, and suboptimal use of inhaler devices is common. ${ }^{4}$ Even in severe asthma where patients are prescribed long-term oral corticosteroids, monitoring of serum drug levels suggests adherence rates of $\sim 50 \% .^{5}$ This may explain why many asthmatics do not achieve control of their disease and why deaths from asthma still occur, often despite identifiable warning signs. ${ }^{6}$ Improving drug management of asthma, like many chronic diseases, needs urgent attention and requires more focus on patients' needs and understanding as well as the use of new technology.

Despite being prescribed high-dose ICS with other controller medications and/or oral corticosteroids (OCS), a proportion of asthmatics $(5 \%-10 \%)$ remain symptomatic 
or have repeated asthma attacks requiring OCS bursts and sometimes hospital admission. ${ }^{7}$ These patients need careful evaluation by specialists to tease out factors contributing to the lack of treatment response. In most series, up to $50 \%$ of apparently uncontrolled asthma is due to nonadherence to therapy, misdiagnosis, and/or coexisting non-asthma pathology. ${ }^{5,8}$ Severe (or refractory) asthma is defined by the International European Respiratory Society/American Thoracic Society guidelines as asthma

[...] which requires treatment with a high dose ICS and a long-acting bronchodilator or leukotriene modifier/ theophylline, for the previous year or systemic corticosteroids for $50 \%$ of the previous year to prevent it from becoming "uncontrolled" or from remaining "uncontrolled" despite this therapy. ${ }^{7}$

This group of patients also accounts for $60 \%$ of all asthmarelated health care costs, including direct drug costs, for outpatient and inpatient care, and represent the group most at risk of suffering from a life-threatening exacerbation. There is a clear need for better identification of severe asthma and for more effective therapies.

An understanding of the immunopathology of asthma has allowed the development of monoclonal antibodies and other biological therapies to address this unmet need in severe asthma, with the emphasis on stratifying this severe or refractory-to-steroid treatment cohort, in order to identify those most likely to benefit from each therapy.

This review focuses on reslizumab - one of the more recently developed biologics; the mechanism by which it exerts its effects and its place in the rapidly expanding arena of biological therapy for asthma is discussed.

\section{Eosinophilic asthma}

Eosinophils are granulocytes characterized by granules containing highly basic proteins, which stain with the acidic dye eosin. They form a minority of blood granulocytes, usually $<300$ per microliter. Since their description by Ehrlich, they have been associated with asthma and allergic diseases, and eosinophil numbers and granule proteins in blood, sputum, bronchoalveolar lavage, or bronchial biopsies have been correlated with asthma severity and increase during spontaneous asthma attacks or asthma provoked experimentally by allergen or viral challenge. ${ }^{9,10}$ Eosinophil granule proteins can damage airway epithelial cells and induce airway hyper-responsiveness (AHR) in animal models, and eosinophils release bronchoconstrictor leukotrienes and proinflammatory cytokines. These observations lead to the "eosinophil hypothesis" of asthma, suggesting that eosinophil activation in the airways was the cause of AHR, mucus hypersecretion, and hence symptoms of asthma. ${ }^{9}$ Of note, eosinophil numbers and eosinophil activation are sensitive to corticosteroids, and successful steroid treatment in asthma was associated with reduction in eosinophils, whereas the so-called "steroid insensitive" asthmatics had persistent eosinophilia despite steroid treatment. ${ }^{11}$ Thus, specific targeting of eosinophils has been an attractive drug target in asthma since at least the 1970s if not the 1870s. It is worth noting that there is some evidence to suggest that a small subset of severe asthma patients may have predominant neutrophilic rather than eosinophilic inflammation. ${ }^{1}$ Whether this reflects a true subtype of asthma or results from superadded infection or the effects of steroid treatment is uncertain. In addition, some other subtypes of asthma have been suggested, including those without persistent inflammation; hence, not all those with severe asthma are candidates for biologics and careful assessment, and selection is essential.

Eosinophils share common lineage with basophils, and both respond in terms of lineage commitment and expansion to the cytokines such as interleukin (IL)-3, IL-5, and granulocyte-macrophage colony-stimulating factor (GM-CSF). ${ }^{9}$ Of these factors, IL-5 is specific to eosinophils and basophils, and particularly in humans, eosinophils seem to be largely dependent on IL-5 for their development in the bone marrow, activation for recruitment to tissues, and survival in sites of inflammation.

The identification of different patterns of cytokine production from subsets of CD4+ T helper lymphocytes in the 1980s raised the possibility that the Th2 subset might be relevant to allergic asthma since it produces IL-4 (which switches B cells to produce immunoglobulin E) and IL-5. ${ }^{12}$ Analysis of cytokine gene expression in the airways of allergic asthmatics showed that this is indeed the case and further that, as in vitro, expression of these cytokines was markedly suppressed by steroids, suggesting an important pathway for the efficacy of steroids in asthma. ${ }^{13,14}$

More recently, it has been shown that other cell types can produce IL-5 and Th2-associated cytokines, including IL-4, IL-9, and IL-13, comprising eosinophils themselves, mast cells, basophils, and innate lymphocytes termed IL-C2. Thus, the cytokine pattern is now usually referred to as type 2 . IL-C2 may be an important initiator and amplifier of eosinophilic inflammation since it responds to alarmin cytokines (thymic stromal lymphopoietin [TSLP], IL-25, and IL-33) produced by epithelial damage. ${ }^{15}$

Over the last 2 decades, specific targeting of these cytokines has been developed through high-affinity monoclonal 
antibodies (and in some cases other molecules) that block the binding of cytokines to their receptors, block the receptors, or deplete cells responding to the cytokines. These biologics are now entering clinics, and one group specifically targets IL-5; that is reslizumab.

\section{Challenges associated with the treatment of eosinophilic asthma and current biological treatment strategies}

As our understanding of the immunopathogenesis of asthma develops, the approach to managing severe asthma is also evolving. The first successful use of a biologic in asthma was that of omalizumab (a monoclonal anti-IgE antibody). ${ }^{16}$ It is effective in patients with severe allergic asthma, resulting in reduced frequency of exacerbation or asthma attacks requiring OCS and a reduction in OCS dose in those patients on longterm OCS treatment. ${ }^{16,17}$ With the development of omalizumab came several important changes in the way we see asthma and assess therapy. First, restriction of this treatment to those with allergic asthma driven by a perennial allergen and total serum IgE in the range of $30-1,500 \mathrm{IU} / \mathrm{mL}$ was the first (perhaps slightly unwitting) step to stratified treatment in severe asthma. At the same time, unbiased cluster-based analysis of clinical features and airway inflammation had identified several subtypes of asthma. ${ }^{18,19}$ It is now accepted that not all types of asthma are the same, and several different endotypes (or inflammatory pathways) may exist. ${ }^{1}$ How much this reflects variable treatment adherence, changes in airway microbial colonization (not in small part due to high-dose ICS), or really represents true subtypes of the underlying disease mechanism remains to be established. Studies such as RASP may answer this point. ${ }^{20}$ It took some time for clinical trials to catch up, but most biologics are now specifically targeted at those asthmatics with biomarkers suggestive of persistent activation of the pathway being blocked by treatment. Broadly speaking, most severe asthmatics can be divided into either allergic, early onset disease (potentially candidates for omalizumab) or late onset, non-allergic, eosinophilic asthma (potentially candidates for anti-IL-5 treatment).

Second, omalizumab studies showed that the major effect was on exacerbation rates rather than on traditional study end points such as lung function. ${ }^{16}$ This has led to exacerbation rates being the primary outcome measure for almost all Phase III studies of biologics in asthma (something we will discuss later).

Selecting eosinophilic patients for anti-IL-5 treatment clearly requires a practical biomarker to identify eosinophilic airway inflammation. Although sputum induction or bronchoscopy may give "gold standard" information on airway inflammation, neither is feasible for use in the clinic. Blood eosinophil counts are probably the most accessible and reliable tool for selecting and assessing patients. Exhaled nitric oxide also correlates with airway eosinophilia, though less well than blood eosinophils. ${ }^{21}$

\section{Comparison of pharmacology and pharmacokinetics of reslizumab}

Reslizumab is a humanized anti-IL-5 IgG4 monoclonal antibody produced by fusing antigen-binding domains of a rat IgG2a anti-IL-5 antibody to human IgG4 kappa chains. ${ }^{22}$ It has a high affinity $\left(K_{\mathrm{d}} 20 \mathrm{pM}\right)$ for the IL-5 binding site of the alpha subunit, acting to neutralize its effect. Reslizumab has a terminal half-life of 25 days, and its effects were maintained for at least 4 weeks after administration with a return to baseline values within 5-6 months.

Likewise, mepolizumab (another monoclonal antibody that has already been approved for use by the US Food and Drug Administration (FDA) and National Institute of Excellence $[\mathrm{NICE}]$ ) binds to IL-5 to prevent it from interacting with its receptor. ${ }^{23}$

IL-5 binds to a receptor consisting of a cytokine-specific alpha chain and a shared beta chain (similar to IL-3 and GMCSF). ${ }^{9}$ Benralizumab (currently being assessed for licensing) is a fully human afucosylated monoclonal antibody that binds to the IL-5 receptor alpha chain and in so doing disrupts signal transduction. The afucosylation confers antibody-dependent cytotoxic properties and thus depletes cell populations expressing IL-5R $\alpha$, namely eosinophils and basophils. ${ }^{24}$

\section{Summary of studies}

Reslizumab has been approved by the FDA and in the UK by NICE.

The initial pilot study conducted by Kips et $\mathrm{al}^{25}$ was a relatively small trial with 32 subjects enrolled in it. It showed that reslizumab was effective in reducing blood and sputum eosinophil counts at a dose of $1 \mathrm{mg} / \mathrm{kg}$ administered intravenously (IV). It was conducted in patients with asthma treated with ICS and/or oral corticosteroids. It did not show a significant effect on forced expiratory volume in the first second of expiration $\left(\mathrm{FEV}_{1}\right), \mathrm{AHR}$, or the airway response to allergen. This study was primarily a safety study and was not powered to detect clinical efficacy. However, in the subgroup of patients with raised eosinophil counts, there was a tendency for these patients to have an increase in $\mathrm{FEV}_{1}$. These findings illustrated several key points: reslizumab is 
safe and effective at reducing eosinophils and, importantly, that it may be necessary to select patients with residual eosinophilia to optimize the effect.

The first large Phase IIb study of reslizumab to stratify patients in this way was by Castro et al. ${ }^{26}$ In a multicentre, randomized, double-blind, placebo-controlled trial $(n=106)$, the clinical efficacy of reslizumab administered IV $(3 \mathrm{mg} / \mathrm{kg}$, 4 weekly) was assessed by comparing Asthma Control Questionnaire (ACQ) scores, eosinophil counts, and lung function in the treatment group versus the placebo group. ${ }^{26}$ Enrolled patients had confirmed airway reactivity, induced eosinophil sputum counts of $\geq 3 \%$, and were on a highdose ICS and a second controller. Reslizumab significantly reduced eosinophil numbers in sputum and improved lung function $\left(\mathrm{FEV}_{1}\right.$ baseline change was $0.18 \mathrm{~L}$ in the treatment group and $-0.08 \mathrm{~L}$ in the placebo group, $p=0.002$ ). ACQ scores showed a trend toward better asthma control in the treatment group, and this was significant in the subgroup analysis of patients with nasal polyps. This study was encouraging as it clearly demonstrated significant benefit in those patients who had refractory eosinophilic asthma.

Two key Phase III multicentre, double-blind, placebocontrolled studies were also published with Castro as the first author. ${ }^{27}$ Patients were selected from $>100$ centers in each study on the basis of a blood eosinophil count of $\geq 400$ per microliter, as this had previously been suggested as a good marker of airway eosinophilia. Screening blood counts could be repeated once, and one eosinophil count $>400$ was sufficient for inclusion. Patients (aged 12-75 years) continued their usual therapy of ICS plus additional controller and OCS of up to $10 \mathrm{mg}$ of prednisolone if used. They were required to be stable for 30 days prior to enrollment, to be symptomatic with an ACQ-7 score of $>1.5$, to have had at least one exacerbation of asthma requiring OCS in the last year, and to have at least $12 \% \mathrm{FEV}_{1}$ reversibility to albuterol to confirm asthma diagnosis. The study included a 2- to 4-week screening run-in then a 52-week treatment phase with either $3 \mathrm{mg} / \mathrm{kg}$ intravenous reslizumab or placebo (randomized in a 1:1 ratio) given every 4 weeks. Patients were stratified by regular OCS use and by region.

The primary outcome for both studies was the frequency of clinical asthma exacerbations (CAE) during the 52-week treatment phase adjudicated by an independent review panel. CAE were defined as increased symptoms requiring OCS or at least a doubling of ICS. Secondary end points included CAE treated with OCS, FEV 1 , ACQ-7 asthma control score, Asthma symptom Utility Index (ASUI) symptom score, Asthma Quality of Life Questionnaire (AQLQ) score, rescue use of shortacting bronchodilators, and blood eosinophil counts.
Frequency of CAE was analyzed by a negative binomial regression model to give rate ratios (RRs) versus placebo, and $\chi^{2}$ tests were used for between-group comparisons.

Of 2,597 patients screened, 1,472 did not meet entry criteria (usually either blood eosinophils were $<400$ or there was insufficient reversibility) and 172 were not included for other reasons.

In study 1, 244 patients had placebo and 245 had infusion of reslizumab, and for study 2 , these numbers were 232 and 232.

There is no mention of systematic assessment of patients or of adherence.

The primary outcome showed a CAE frequency of 1.81 per patient per year for placebo and 0.90 for reslizumab treatment in study 1 and 2.11 versus 0.86 in study 2 (statistically significant at $p<0.0001$ for both studies with RR values of 0.50 and 0.41 , respectively).

This reduction in exacerbation frequency was also seen for exacerbations requiring OCS (which was a majority of exacerbations), and there were significant increases in $\mathrm{FEV}_{1}$, ACQ-7, ASUI, and AQLQ with reslizumab treatment, reduction in rescue beta 2 agonist puffs taken, and falls in blood eosinophils. The Kaplan-Meier plot from one of the two studies, for frequency of remaining exacerbation free, is shown in Figure 1A. Although hospitalization rates were reduced, this was a rare outcome, and the difference was not statistically significant: 57 admissions on placebo versus 33 on active treatment were estimated. Hospital admission was much rarer in study 2 than in study 1 . In study $1,44 \%$ of patients on placebo were exacerbation free over the year of the trial versus $61 \%$ with reslizumab, and in study 2 , these percentages were 52 and 73 , respectively.

There were quite large placebo effects for $\mathrm{FEV}_{1}$ (Figure 1B) and AQLQ, but less so for exacerbation frequency, where baseline for placebo was 2.1/2.0 compared to CAE rates of 1.80 and 2.11 over the 52 weeks of each study.

The drug was well tolerated with few local infusion reactions $(<3 \%$; no difference existed between the drug and placebo). Two reslizumab-treated patients had anaphylaxis; it is stated that these patients responded to standard treatment, though not whether epinephrine was required. Both patients were withdrawn from the study, and were negative for antidrug antibodies. An anaphylaxis rate of just less than 1 in 200 patients was observed. In total, 15 patients treated with reslizumab developed antidrug antibodies, but none were neutralizing and no adverse effects were seen in these subjects.

Because patients were offered open-label extension, incomplete data were available on the effect of stopping treatment, 

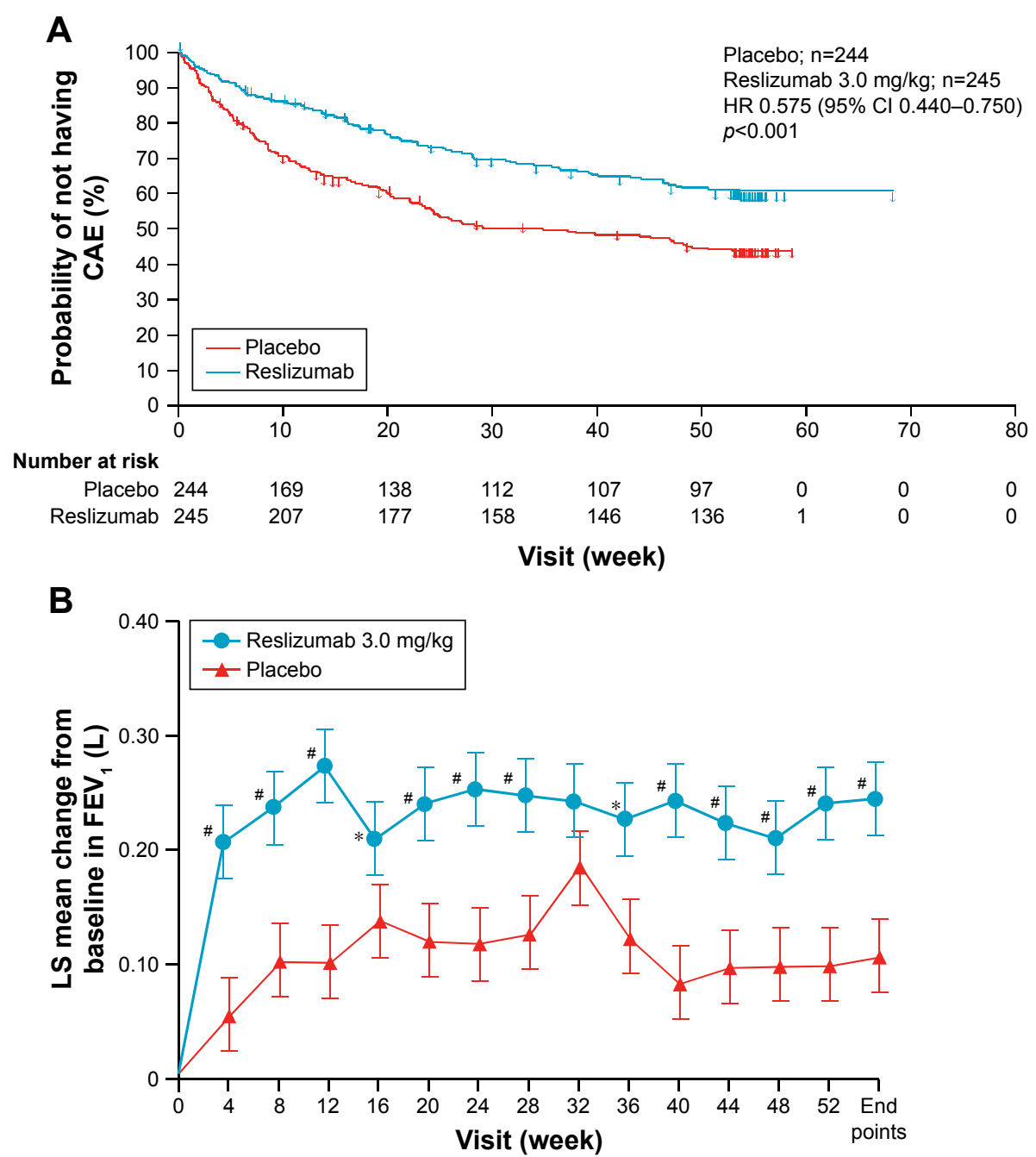

Figure I Effect of reslizumab versus placebo on exacerbation frequency and $\mathrm{FEV}_{1}$.

Notes: (A) Kaplan-Meier plot showing frequency versus time for remaining exacerbation free in patients treated with reslizumab or placebo for one of the two Phase III studies. (B) Change in FEV, over time for patients treated with reslizumab or placebo in one of the two Phase III studies. *p<0.05. \# $<<0.0$ I. Reprinted from Lancet Respir Med, 20I5;3:355-366. Castro M, Zangrilli J, Wechsler ME, et al. Reslizumab for inadequately controlled asthma with elevated blood eosinophil counts: results from two multicentre, parallel, double-blind, randomised, placebo-controlled, phase 3 trials. Copyright (20I5), with permission from Elsevier. ${ }^{27}$

Abbreviations: $\mathrm{CAE}$, clinical asthma exacerbations; $\mathrm{FEV}_{1}$, forced expiratory volume in the first second of expiration; HR, hazard ratio; LS, least squares.

but eosinophil counts were back to baseline by 4 months after treatment in the 75 patients who did not continue the drug.

Subsequent post hoc analysis of the two Phase III studies by Brusselle et al, suggest that late onset asthma (after the age of 40 years) responded better than early onset disease, even though $41 \%$ of the late onset group was atopic compared to $69 \%$ of the early onset patients. ${ }^{28}$ None of these patients had previously been treated with omalizumab.

Subsequently, Corren et $\mathrm{al}^{29}$ also confirmed that reslizumab is clinically efficacious in patients with an eosinophilia count $>400$ cells $/ \mu \mathrm{L}$. The study $(\mathrm{n}=496)$ recruited patients on at least a medium-dose ICS \pm long-acting beta agonist, unselected for baseline eosinophilia but confirmed airway reversibility of $\geq 12 \%$ following administration of a short-acting beta agonist. This study showed an improvement in pulmonary function, ACQ scores, and rescue inhaler use in the subgroup analysis of patients with $\geq 400$ cells $/ \mu \mathrm{L}$; however, interpretation is limited as the study was not designed or statistically powered to test this group ( $n=69$ in the treatment arm versus $n=13$ in placebo). The study appears to have been conducted to confirm that reslizumab has no significant effect if blood eosinophils are $<400$ cells $/ \mu \mathrm{L}$, although differences between groups were not statistically significant. One of 398 patients receiving reslizumab had anaphylaxis.

Bjermer et $\mathrm{al}^{30}$ reported the efficacy and safety of reslizumab in a dose-comparison study over 16 weeks with $\mathrm{FEV}_{1}$ as the primary outcome. Patients were randomized to receive either 0.3 or $3.0 \mathrm{mg} / \mathrm{kg}$ of reslizumab or placebo, with just over 100 per group. Effect sizes were larger in the $3.0 \mathrm{mg} / \mathrm{kg}$ group (eg, for $\mathrm{FEV}_{1}$, the difference versus placebo was 
$0.115 \mathrm{~L}$ for the $0.3 \mathrm{mg} / \mathrm{kg}$ dose and $0.160 \mathrm{~L}$ for the $3.0 \mathrm{mg} / \mathrm{kg}$ dose, though it is not stated if this was a significant change in the difference). This trial concluded that $3.0 \mathrm{mg} / \mathrm{kg}$ was the most effective dose with a minimal side effect profile. No anaphylaxis was reported. Again, the baseline characteristics of patients were refractory asthma and a blood eosinophil count of $\geq 400$ cells $/ \mu \mathrm{L}$.

In summary, these studies show that reslizumab is most effective in patients with severe asthma and an eosinophil count of $\geq 400$ cells $/ \mu \mathrm{L}$. Furthermore, a dose of $3.0 \mathrm{mg} / \mathrm{kg}$ IV showed the most pronounced effect without an increase in adverse events (AEs).

\section{Safety, efficacy, and tolerability of reslizumab compared with other biologically based therapies for eosinophilic asthma}

Reslizumab has consistently demonstrated that it is safe and well tolerated, with similar or fewer AEs compared to the respective placebo arms of the trial. The clinical trials encompassed the age range of 12-75 years between them. Most common AEs included headache, worsening of asthma symptoms, upper respiratory tract infections, and nasopharyngitis. Indeed, there were fewer infections in the treatment arm compared to placebo. In addition, patients were less likely to discontinue treatment due to AEs in the reslizumab group.

Murphy et $\mathrm{al}^{31}$ presented data in 2015 looking at longterm safety and efficacy of reslizumab over a period of 2 years as part of an open-label extension study following the Phase III trials summarized earlier. In total, 1,052 patients were enrolled: 572 patients in the reslizumab-experienced group and 480 patients in the reslizumab-naïve group. Again, reslizumab was safe and well tolerated, with the incidence of AEs similar across the two groups: there were no parasitic or helminthic infections, and the frequency of malignancy reflected that of the general population. There were no instances of anaphylaxis.

The longer-term sequelae are still relatively unknown as is its safety in pregnancy.

\section{Comparison to other anti-IL-5 agents}

Comparisons are inevitable with similar agents recently approved or currently in development. Mepolizumab like reslizumab targets IL-5 and prevents it from binding to its receptor. ${ }^{2,3}$ It is available in a 4 weekly dosing regime of $100 \mathrm{mg}$ subcutaneously. To date, there has been no direct head-to-head studies comparing reslizumab and mepolizumab. Mepolizumab is also very well tolerated with a favorable safety profile. It has been approved by the FDA and European regulators. It is licensed for use in those patients with refractory eosinophilic asthma. Studies included patients with eosinophil counts either $\geq 150$ cells $/ \mu \mathrm{L}$ at screening or $>300$ cells $/ \mu \mathrm{L}$ in the past year. ${ }^{32,33}$ Studies have shown that it reduced exacerbations, has a steroid sparing effect, improved lung function, and improved quality of life measures. ${ }^{32-34}$ Furthermore, as it is a subcutaneous formulation, it is more convenient and acceptable to the patient and less time-consuming.

Benralizumab appears to be effective in reducing both tissue and serum eosinophils rapidly, and there may be a role for its use in emergency presentations. As IL-5 receptors are expressed on eosinophils, eosinophil progenitors, and basophils, it will affect all these populations. Again, Phase III studies showed an approximately 50\% reduction in exacerbation rates and effects on lung function, ACQ, and quality of life. ${ }^{35,36}$ Benralizumab is given every 8 weeks by subcutaneous injection.

From the trial data, it is not possible to recommend one anti-IL-5 agent over another: all seem to be effective at reducing exacerbation rates in severe eosinophilic asthma. It was a surprise to many that the low dose of mepolizumab was as effective as higher doses in the DREAM study, ${ }^{33}$ particularly since this dose does not reduce airway eosinophil numbers, at least in short-term studies. The lack of superiority of benralizumab was also of interest since this antibody does reduce eosinophils in tissue rapidly and dramatically. The results might suggest that the effect of these agents is to reduce the bone marrow pool of eosinophils available for mobilization and thus reduce eosinophilic asthma attacks. Clearly, some exacerbations do still occur in patients treated with antiIL-5 monoclonal antibodies. We need to know whether this is a breakthrough for eosinophilia or, more likely, whether another mechanism is at play. Importantly, we need to know whether such exacerbations are OCS responsive, since a major role of OCS is to reduce eosinophils.

These drugs are expensive. In the UK, the NICE assesses all new drugs, and they are most concerned about costeffectiveness. The approved biologics for asthma have all had to cut a deal on price to get NICE approval based on cost per quality-adjusted life year (QUALY) gained. There is quite wide geographical variation in drug price for these agents. As more and more biologics are available for asthma and other chronic diseases (possibly including the anti-IL-5 biologics for $\mathrm{COPD}^{37}$ ), there will be more pressure from 
health payers to reduce costs. With this will come reduced returns as more drugs chase a defined patient population.

For anti-IL-5 drugs, these considerations are seen in the stringent criteria set by UK authorities for their use. For mepolizumab, there is a requirement for assessment of asthma at a designated severe asthma center, severe asthma defined by treatment at step 4 or 5 of guidelines, at least four exacerbations requiring OCS in the last year, and a blood eosinophil count of $\geq 300$ per microliter in the last year (and of course the undisclosed NHS discount price)..$^{38}$ For reslizumab, the criteria (still in development at the time of writing) are slightly different: three exacerbations in the last year and an eosinophil count of $\geq 400 \mu \mathrm{L}$. $^{39}$

At present, we do not have any data to suggest that reslizumab is more effective than mepolizumab. There will be a few patients who have had three but not four exacerbations in the last year. Otherwise, it seems unlikely that physicians or patients will choose an intravenous drug over a subcutaneous one. In other countries, price might be a determining feature, but this remains unclear.

One distinguishing feature of mepolizumab over reslizumab is that mepolizumab has a confirmed OCS-sparing effect, with a halving of OCS dose seen in the SIRIUS study ${ }^{34}$ a similar effect was reported for benralizumab. ${ }^{40}$ Long-term OCS have many side-effects which can make patients reluctant to take regularly and a concern for doctors who want to avoid these complications. We need information on the OCS-sparing effect of both reslizumab and mepolizumab, and it is a surprise that the regulators did not ask for this. However, we should also be phlegmatic and ask just how clinically relevant reducing from 10 to $5 \mathrm{mg}$ of prednisolone daily is for long-term side effects in severe asthma (at present, we just do not know).

The next issue for consideration is the stopping rules. There can be no justification in spending thousands of pounds on treatments that are not working. How do we assess efficacy in individual patients? For omalizumab, there is a generally agreed (if perhaps not very scientific) 16-week assessment. Patients not responding to omalizumab could be switched to anti-IL-5 treatment.

For mepolizumab, it is suggested that treatment is given for 1 year and exacerbation reduction assessed. ${ }^{38}$

Reslizumab had effects on $\mathrm{FEV}_{1}$ and other outcomes by week 4; we need to know whether there are markers that can be used to select responders early in treatment.

There seems no logic for switching from one anti-IL-5 agent to another if a patient fails to respond. However, some information to confirm this would be of interest.
At present, there are little data on long-term efficacy of biologic treatment for asthma. There seems no reason why these drugs should "reset" asthma so that they can be discontinued. One study of omalizumab did suggest that $\sim 50 \%$ of patients who discontinued omalizumab after long-term (5 years plus) therapy remained exacerbation free for a year. ${ }^{41}$ We need to know why. It is noteworthy that half of the patients in the reslizumab Phase III studies did not have exacerbations over a 1 -year period even on placebo. ${ }^{27}$ Treatment may therefore result in reversion of disease in selected patients who have frequent exacerbations; we need information on whether this represents a stable long-term "phenotype".

As mentioned earlier, approximately half of the patients with severe persistent asthma are non-adherent to treatment. As inhaler-monitoring technology develops, it is clear that our traditional approaches to assessing adherence are less than adequate; it will be of great interest to see if monitoring devices improve on this. ${ }^{42}$ We need to make it much easier to scrutinize prescription records to see if a patient was prescribed, and picked up, regular preventer inhalers. We should assess inhaler use at every visit and use monitors to assess and improve adherence. We would argue that companies that spend hundreds of millions of dollars (pounds, euros, etc.) developing biologics should pay more attention to rigorous assessment of asthmatics, including adherence (and we think they are beginning to).

Reslizumab was associated with anaphylaxis in a small number of cases, and this is also described for omalizumab. This raises the issue of whether these agents need to be given in hospital or may be suitable for self-injection (as for some biologics in rheumatoid arthritis). It will be important to establish safety and efficacy of such an approach before it is used widely in clinical practice.

\section{Conclusion and future directions}

Reslizumab is an anti-IL-5 monoclonal antibody that reduced exacerbation rates by $50 \%$ in Phase III studies, with accompanying improvements in lung function, asthma control, and quality of life.

At present, it adds little over mepolizumab, which has similar effects (Table 1), but is given by subcutaneous route and has documented OCS-sparing activity.

As a further IL-5-directed biologic, benralizumab (Table 1) becomes available, these agents can be combined with other biologics, including duplimab (which blocks IL-4 and IL-13) ${ }^{43}$ and tezepelumab (targeting TSLP), that show promise in large Phase II studies. ${ }^{44}$ In addition, small molecules, such as fevipiprant, which block prostaglandin D2 receptors may compete in type 2 severe asthma. ${ }^{45}$ 
Table I Phase III data for biologics targeting IL-5

\begin{tabular}{|c|c|c|c|}
\hline Parameter & Reslizumab & Mepolizumab & Benralizumab \\
\hline Target & IL-5 & IL-5 & IL-5R $\alpha$ \\
\hline \multicolumn{4}{|l|}{ Inclusions } \\
\hline Blood eos & $\geq 400$ & $\geq 150^{\mathrm{a}}$ & $\geq 300$ \\
\hline Exacerbations & $\geq 1$ & $\geq 2$ & $\geq 2$ \\
\hline Numbers & 953 & $385^{a}$ & $482 / 534$ \\
\hline Dosing interval & $4 w$ & $4 w$ & $8 w$ \\
\hline Route & iv & sc & sc \\
\hline \multicolumn{4}{|l|}{ Outcomes } \\
\hline \multicolumn{4}{|l|}{ Exacerbations/year } \\
\hline Baseline & 2.0 & 3.6 & $2.7 / 3.0$ \\
\hline Placebo & 1.81 & 1.74 & $0.93 / 1.33$ \\
\hline Active & 0.84 & 0.83 & $0.66 / 0.65$ \\
\hline $\mathrm{RR}$ & 0.46 & 0.53 & $0.72 / 0.49$ \\
\hline \multicolumn{4}{|l|}{ Change in $\mathrm{FEV}_{1}(\mathrm{~L})$} \\
\hline Placebo & 0.120 & 0.086 & $0.210 / 0.239$ \\
\hline Active & 0.220 & 0.183 & $0.330 / 0.398$ \\
\hline \multicolumn{4}{|l|}{ Change in ACQ-7 } \\
\hline Placebo & -0.77 & -0.50 & $-1.19 /-1.17^{b}$ \\
\hline Active & -1.02 & -0.94 & $-1.44 /-1.46$ \\
\hline Oral steroid-sparing effect & - & $50 \%$ & $75 \%{ }^{\mathrm{b}}$ \\
\hline Anaphylaxis & 2 & 0 & $4 / 2^{c}$ \\
\hline
\end{tabular}

Notes: anclusion criterion was 150 eosinophils per microliter of blood at screening or 300 eosinophils per microliter of blood in the previous year; 385 was the number of patients treated with either placebo or subcutaneous mepolizumab (there was also an iv arm). ${ }^{\text {bCQ }}-6$ was used in the benralizumab Phase III trials. There was a $25 \%$ OCS reduction in the placebo group in the benralizumab study (no reduction with placebo was seen in the mepolizumab trial). 'Reported as "hypersensitivity reactions". Refs, reslizumab, ${ }^{27}$ mepolizumab, ${ }^{32}$ and $^{34}$ benralizumab. ${ }^{35,36,40}$

Abbreviations: IL, interleukin; eos, eosinophils; w, weekly; iv, intravenous; sc, subcutaneous; RR, relative risk; FEV , forced expiratory volume at I second; ACQ, asthma control questionnaire; OCS, oral corticosteroids.

Thus, biologic options for severe asthma are here, expanding, and welcome! We need well-directed use of these agents and more information on patient selection, monitoring outcomes, and long-term effects so that we can get the best asthma therapy for our patients from these exciting new opportunities.

\section{Disclosure}

The authors report no conflicts of interest in this work.

\section{References}

1. Pavord ID, Beasley R, Agusti A, et al. After asthma: redefining airways diseases. Lancet. Epub 2017 Sep 11.

2. GINA [webpage on the Internet]. 2017 GINA Report, Global Strategy for Asthma Management and Prevention. Available from: http://ginasthma. org/2017-gina-report-global-strategy-for-asthma-management-andprevention. Accessed January 31, 2018.

3. British Thoracic Society [webpage on the Internet]. BTS/SIGN British guideline on the management of asthma. Available from: https://www.brit-thoracic.org.uk/standards-of-care/guidelines/ btssign-british-guideline-on-the-management-of-asthma. Accessed January 31, 2018.

4. Lindsay JT, Heaney LG. Non-adherence in difficult asthma and advances in detection. Exp Rev Respir Med. 2013;7(6):607-614.

5. Robinson DS, Campbell DA, Durham SR, Pfeffer J, Barnes PJ, Chung KF. Systematic assessment of difficult-to-treat asthma. Eur Respir J. 2003; 22(3):478-483.
6. RCP. Why asthma still kills: the National Review of Asthma Deaths (NRAD) Confidential Enquiry report, 2014. Available from: http:// www.rcplondon.ac.uk/sites/default/files/why-asthma-still-kills-fullreport.pdf. Accessed January 31, 2018.

7. Chung KF, Wenzel SE, BrozekJL, et al. International ERS/ATS guidelines on definition, evaluation and treatment of severe asthma. Eur Respir J. 2014;43(2):343-373.

8. Robinson DS. Assessing severe asthma. Eur Respir J. 2016;48: 611-613.

9. Kariyawasam HH, Robinson DS. The eosinophil: the cell and its weapons, the cytokines, its locations. Semin Respir Crit Care Med. 2006;27:117-127.

10. Bousquet J, Chanez P, Lacoste JY, et al. Eosinophilic inflammation in asthma. N Engl J Med. 1990;323:1033-1039.

11. Leung DY, Martin RJ, Szefler SJ, et al. Dysregulation of interleukin 4, interleukin 5, and interferon gamma gene expression in steroid-resistant asthma. J Exp Med. 1995;181:33-40.

12. Mosmann TR, Cherwinski H, Bond MW, Giedlin MA, Coffman RL. Two types of murine helper T cell clone. I. Definition according to profiles of lymphokine activities and secreted proteins. JImmunol. 1986; 136:2348-2357.

13. Robinson DS, Hamid Q, Ying S, et al. Predominant TH2-like bronchoalveolar T-lymphocyte population in atopic asthma. $N$ Engl J Med. 1992;326:298-304.

14. Robinson D, Hamid Q, Ying S, et al. Prednisolone treatment in asthma is associated with modulation of bronchoalveolar lavage cell interleukin-4, interleukin-5, and interferon-gamma cytokine gene expression. Am Rev Respir Dis. 1993;148:401-406.

15. Robinson D, Humbert M, Buhl R, et al. Revisiting type 2-high and type 2-low airway inflammation in asthma: current knowledge and therapeutic implications. Clin Exp Allergy. 2017;47:161-175. 
16. Humbert M, Beasley R, Ayres J, et al. Benefits of omalizumab as add-on therapy in patients with severe persistent asthma who are inadequately controlled despite best available therapy (GINA 2002 step 4 treatment): INNOVATE. Allergy. 2005;60:309-316.

17. Humbert M, Busse W, Hanania NA, et al. Omalizumab in asthma: an update on recent developments. J Allergy Clin Immunol Pract. 2014; 2:525-536

18. Haldar P, Pavord ID, Shaw DE, et al. Cluster analysis and clinical asthma phenotypes. Am J Respir Crit Care Med. 2008;178:218-224.

19. Moore WC, Meyers DA, Wenzel SE, et al. Identification of asthma phenotypes using cluster analysis in the Severe Asthma Research Program. Am J Respir Crit Care Med. 2010;181:315-323.

20. Heaney LG, Djukanovic R, Woodcock A, et al. Research in progress: Medical Research Council United Kingdom Refractory Asthma Stratification Programme (RASP-UK). Thorax. 2016;71:187-189.

21. Coumou H, Bel EH. Improving the diagnosis of eosinophilic asthma. Expert Rev Respir Med. 2016;10:1093-1103.

22. Egan RW, Athwal D, Bodmer MW, et al. Effect of Sch 55700, a humanized monoclonal antibody to human interleukin-5, on eosinophilic responses and bronchial hyperreactivity. Arzneimittelforschung. 1999; 49:779-790.

23. Robinson DS. Mepolizumab treatment for asthma. Expert Opin Biol Ther. 2013;13:295-302.

24. Molfino NA, Gossage D, Kolbeck R, Parker JM, Geba GP. Molecular and clinical rationale for therapeutic targeting of interleukin-5 and its receptor. Clin Exp Allergy. 2012;42:712-737.

25. Kips JC, O'Connor BJ, Langley SJ, et al. Effect of SCH55700, a humanized anti-human interleukin-5 antibody, in severe persistent asthma: A pilot study. Am J Respir Crit Care Med. 2003;167:1655-1659.

26. Castro M, Mathur S, Hargreave F, et al. Reslizumab for poorly controlled, eosinophilic asthma. Am J Respir Crit Care Med. 2011;184: 1125-1132.

27. Castro M, Zangrilli J, Wechsler ME, et al. Reslizumab for inadequately controlled asthma with elevated blood eosinophil counts: results from two multicentre, parallel, double-blind, randomised, placebo-controlled, phase 3 trials. Lancet Respir Med. 2015;3:355-366.

28. Brusselle G, Germinaro M, Weiss S, Zangrilli J. Reslizumab in patients with inadequately controlled late-onset asthma and elevated blood eosinophils. Pulm Phamacol Ther. 2017;43:39-45.

29. Corren J, Weinstein S, Janka L, Zangrilli J, Garin M. Phase 3 study of reslizumab in patients with poorly controlled asthma: effects across a broad range of eosinophil counts. Chest. 2016;150:799-810.

30. Bjermer L, Lemiere C, Maspero J, Weiss S, Zangrilli J, Germinaro M. Reslizumab for inadequately controlled asthma with elevated blood eosinophil levels: a randomized phase 3 study. Chest. 2016;150: 789-798.

31. Murphy K, Jacobs J, Bjermer L, Shalit Y, Garin M. Long-term safety and efficacy of reslizumab in patients with inadequately controlled, moderate-to-severe asthma and elevated blood eosinophil counts: an open-label extension study. 2017;5(6):1572-1581.
32. Ortega HG, Liu MC, Pavord ID, et al. Mepolizumab treatment in patients with severe eosinophilic asthma. $N$ Engl J Med. 2014;371: 1198-1207.

33. Pavord ID, Korn S, Howarth P, et al. Mepolizumab for severe eosinophilic asthma (DREAM): a multicentre, double-blind, placebocontrolled trial. Lancet. 2012;380:651-659.

34. Bel EH, Wenzel SE, Thompson PJ, et al; SIRIUS Investigators. Oral glucocorticoid-sparing effect of mepolizumab in eosinophilic asthma. N Engl J Med. 2014;371:1189-1197.

35. Bleecker ER, FitzGerald JM, Chanez P, et al. Efficacy and safety of benralizumab for patients with severe asthma uncontrolled with highdosage inhaled corticosteroids and long-acting $\beta 2$-agonists (SIROCCO): a randomised, multicentre, placebo-controlled phase 3 trial. Lancet. 2016;388:2115-2127.

36. FitzGerald JM, Bleecker ER, Nair P, et al. Benralizumab, an antiinterleukin-5 receptor $\alpha$ monoclonal antibody, as add-on treatment for patients with severe, uncontrolled, eosinophilic asthma (CALIMA): a randomised, double-blind, placebo-controlled phase 3 trial. Lancet. 2016;388:2128-2141.

37. Pavord ID, Chanez P, Criner GJ, et al. Mepolizumab for eosinophilic chronic obstructive pulmonary disease. N Engl J Med. 2017;377: 1613-1629.

38. NICE [webpage on the Internet]. Mepolizumab for treating severe refractory eosinophilic asthma. Available from: https://www.nice.org. uk/guidance/ta431. Accessed January 31, 2018.

39. NICE. [webpage on the Internet]. Reslizumab for treating severe eosinophilic asthma. Available from: https:/www.nice.org.uk/guidance/ta479. Accessed March 3, 2018.

40. Nair P, Wenzel S, Rabe KF, et al. Oral Glucocorticoid-Sparing Effect of Benralizumab in Severe Asthma. N Engl J Med. 2017;376: 2448-2458.

41. Ledford D, Busse W, Trzaskoma B, et al. A randomized multicenter study evaluating Xolair persistence of response after long-term therapy. J Allergy Clin Immunol. 2017;140:162-169.

42. Mokoka MC, Lombard L, MacHale EM, et al. In patients with severe uncontrolled asthma, does knowledge of adherence and inhaler technique using electronic monitoring improve clinical decision making? A protocol for a randomised controlled trial. BMJ Open. 2017;7(6): e015367.

43. Wenzel S, Castro M, Corren J, et al. Dupilumab efficacy and safety in adults with uncontrolled persistent asthma despite use of mediumto-high-dose inhaled corticosteroids plus a long-acting $\beta 2$ agonist: a randomised double-blind placebo-controlled pivotal phase $2 \mathrm{~b}$ doseranging trial. Lancet. 2016;388:31-44.

44. Corren J, Parnes JR, Wang L, et al. Tezepelumab in adults with uncontrolled asthma. N Engl J Med. 2017;377:936-946.

45. Gonem S, Berair R, Singapuri A, et al. Fevipiprant, a prostaglandin D2 receptor 2 antagonist, in patients with persistent eosinophilic asthma: a single-centre, randomised, double-blind, parallel-group, placebocontrolled trial. Lancet Respir Med. 2016;4:699-707.

\section{Publish your work in this journal}

Drug Design, Development and Therapy is an international, peerreviewed open-access journal that spans the spectrum of drug design and development through to clinical applications. Clinical outcomes, patient safety, and programs for the development and effective, safe, and sustained use of medicines are the features of the journal, which

\section{Dovepress}

has also been accepted for indexing on PubMed Central. The manuscript management system is completely online and includes a very quick and fair peer-review system, which is all easy to use. Visit http://www.dovepress.com/testimonials.php to read real quotes from published authors. 